\title{
Racionalidad de la inducción como minimización entrópica
}

Rationality of induction as entropic minimization

\author{
IGNACIO SOLS \\ Universidad Complutense \\ isols@mat.ucm.es
}

En recuerdo de D. Mariano Artigas

Resumen. En apoyo de un aspecto fundamental de la epistemología de Mariano Artigas - la intención de verdad en la teoría científica- argumento a favor de la racionalidad del proceso de inducción, tanto de la inducción de leyes generales a partir de hechos de experiencia particulares, como de la inferencia de postulados a partir de las leyes experimentales que constituyen la base empírica de la teoría. Para ello muestro que, al inducir, minimizamos la entropía de la información de que disponemos, y que por tanto hacemos lo que hace siempre la razón.

Palabras clave: inducción; postulados; teoría científica; realismo; entropía algorítmica.

Abstract. In favour of a fundamental aspect of the epistemology of Mariano Artigas -his commitment to the quest for truth on the part of the scientific theory- I argue about the rationality of the process of induction, both the induction of general laws from particular facts of experience and the inference of postulates from the experimental laws which form the empirical basis of the theory. This is argued by showing that we minimize the entropy of the information when making an induction, so that we just do what usually the reason does.

Keywords: induction; postulates; scientific theory; realism; algorithmic entropy. 
D. Mariano. Así le llamábamos todos, y así le queríamos. Mi deuda humana con él es impagable, pero no puedo ni debo hablar de esto aquí -“secretum meum mihi" - sino más bien de la deuda intelectual que tengo, como muchos de nosotros, con D. Mariano. En mi caso, es tanto por lo que directamente aprendí de él como por lo que después leí en su obra publicada. D. Mariano pensó y enseñó a pensar. A él debo mi inclinación a la filosofía. Como contribución al homenaje al maestro, intentaré prolongar dos pinceladas que quedaron esbozadas en su obra: la justificación de la inducción de leyes universales y la de la inferencia, desde estas leyes, de los postulados de la teoría. La racionalidad de ambos procesos será explicada en términos de minimización de entropía. Las dos implicaciones que en este artículo intento remontar -ascender de implicado a implicante-, son pues las siguientes:

postulados $\rightarrow$ leyes experimentales $\rightarrow$ hechos de experiencia $(p \rightarrow l \rightarrow h)$

No se trata ciertamente de remontar estas implicaciones con ayuda de la lógica, pues esta nunca llevará de implicado a implicante, pero sí con ayuda de la razón, la cual es algo más que la mera lógica.

\section{Dos justificaciones pendientes}

Hay un acierto evidente en la obra de Mariano Artigas - acierto que vacuna contra el positivismo- al caracterizar la ciencia experimental como aquella que construye su propio objeto ("caracterizar" es algo más atrevido y sustancial que el mero "demarcar" popperiano). El lector de su obra que no reflexiona lo suficiente sobre su significado profundo puede pensar que su concepción de la construcción del objeto - al incluir los postulados entre las estipulaciones de la teoría que construyen el objeto bajo estudio- está abocada a un convencionalismo que pondría la forma en el método. Tal convencionalismo sería ciego a la forma existente en la realidad natural más bien apriorismo kantiano que hilemorfismo aristotélico-, y es nuestro primer objetivo mostrar que no es en realidad así1.

1 Sobre la necesidad de una inyección de hilemorfismo en la actual epistemología científica merece ser oída la voz de Rudolf Larenz en algunos de sus recientes artículos, y en este 
Además, muchos de los epistemólogos actuales evitan el espinoso tema de la justificación racional de la inducción, o niegan su validez por el hecho de que la lógica impide ir de implicado a implicante (en lo que olvidan lo que va a ser Leit Motiv de este artículo: la razón es más que la lógica). Mariano Artigas no evita este tema, sino que se pronuncia en múltiples lugares de su obra a favor de la validez de la inducción. Mi segunda contribución será un intento de justificación de esta validez, viendo la inducción como un proceso que minimiza la entropía de la información. Argumentaré que, por eso mismo, se trata de un proceso racional.

Comenzaré recordando lo obvio, pero que a veces se olvida: que en cuestión de epistemología, son tareas muy distintas la descripción del método científico y la discusión de su fiabilidad. Lo primero no parece un discurso propiamente filosófico, aunque tampoco lo es científico, pues, obviamente, la descripción del método de una ciencia no es parte de esa ciencia. En realidad, no es conocimiento propiamente dicho, sino mera descripción de los modos de hacer admitidos (al menos implícitamente) por la comunidad científica, como modo de ampliación del conocimiento. No es de extrañar, pues, que en el primer aspecto haya cierto acuerdo, independiente de las diversas posturas filosóficas. Incluso en el caso en que parece haber desacuerdo - Popper versus Kuhn y aún más versus Feyerabend-, no es difícil mostrar que ese desacuerdo es solo superficial.

Observa Hegel, en su Introducción a la Historia de la Filosofía, que la historia implementa siempre las derivaciones que estaban implícitas en la idea, o sea, las implicaciones lógicas que el pensador no se ha atrevido a extraer de sus propias ideas, pero que inexorablemente extraerán su seguidores. En consecuencia, según Hegel, la historia del pensamiento describe a grandes rasgos, y desplegada en el tiempo, la lógica que entrelaza las ideas mismas. Con esta visión in mente, la descripción del método científico en términos sociológicos por Thomas Kuhn -incluso la descripción ácrata de Paul Feyerabend - no resulta tan distinta de la descripción lógica de Karl Popper, que da nombre a su obra más influyente. En efecto, el proceso lógico de falsación

sentido es también muy bienvenido el esfuerzo de recuperación de la forma -forma en el ser, forma como causa fundante del ser- en la epistemología reciente de Juan Arana. 
que Popper describe con rigor, coincide con el que Kuhn registra en la historia de la ciencia como "cambio de paradigma", limitándose la observación de Kuhn al hecho evidente de que la falsación no suele producirse en la biografía de un científico - que suele resistirse, ante la experiencia anómala, a dar por falsa la teoría en que siempre ha creído- pero sí se produce en la biografía de la ciencia, puesto que esa experiencia anómala, o conjunto de experiencias anómalas, terminan por dar paso a la nueva teoría ${ }^{2}$.En cuanto a Paul Feyerabend, cabe decir lo mismo: líbrese a la ciencia del corsé de las reglas metodológicas tal como él recomienda, y acabará cumpliendo todas las reglas que la hubieran encorsetado: acabará evolucionando, a la larga, siguiendo el implacable proceso lógico descrito por Karl Popper.

Basado, pues, en que hay cierto acuerdo en las diversas descripciones del método científico, me permito comenzar con un breve recuerdo de este método, antes de pasar a la discusión de su fiabilidad. En particular, parece acertado el recurso que Evandro Agazzi y Mariano Artigas hacen del operacionismo como fuente de la intersubjetividad - o apreciación unánime de los científicos - de las experiencias que fundamentan el edificio de la ciencia: yo no sé si el color rojo que otro percibe es tal como lo percibo yo, pero podemos volver esta percepción intersubjetiva conviniendo cierta “operación”. Por ejemplo, que ambos pulsemos un botón cada vez que sea roja la luz que se enciende en un panel. Mediante protocolos y operaciones de algún modo convenidos logramos objetivar una parcela de la realidad que se convierte entonces en objeto para que una determinada ciencia lo pueda estudiar. De hecho, parece acertado el modo en que Mariano Artigas caracteriza la ciencia experimental como aquella que, de este modo "operacional”, crea su propio objeto de estudio. En esta primera fase del método científico - la creación de la base experimental de la teoría-, se llegan a formular leyes generales mediante inducción. Cuando la naturaleza muestra siempre un

2 Como ejemplo ilustrativo, cabe citar la experiencia de Michelson-Morley. No se sabe siquiera si Einstein tenía conocimiento de ella, por lo que no tiene sentido decir que esta experiencia mostró falsa la mecánica clásica en la mente de Einstein, y, sin embargo, no cabe duda de que la mecánica clásica quedó falsada por ella en la mente de los científicos, puesto que la nueva teoría de Einstein no se hubiese abierto camino sin esa experiencia anómala, y otras que le siguieron. 
mismo comportamiento - dentro del nivel de aproximación que permiten los instrumentos utilizados-, y esto ocurre en repetidos experimentos bajo las mismas condiciones, "inducimos" la ley general que expresa esa regularidad de la naturaleza mediante relaciones matemáticas entre las magnitudes medidas con los aparatos y unidades convenidos. Como P. Duhem observa, el registro de los resultados de experiencia ha de ser siempre en simbolismo matemático: nada significa para la ciencia una "distancia larga”, pero sí significa que una distancia sea el doble o triple que otra. Hasta que la convención de una unidad no haya convertido el resultado de las experiencias en número, no pueden gozar de la intersubjetividad de la matemática. Solo las relaciones entre magnitudes son matematizables, y por tanto solo ellas pueden ser intersubjetivas.

Llegamos así a la segunda fase de la construcción de la teoría científica. Acabada esa primera de establecimiento de su base experimental o conjunto de leyes universales inducido a partir de hechos experimentados -o llevada al menos a cierta madurez-, se proponen -se “inducen”, o más propiamente se "infieren"- en términos no necesariamente observables (pero siempre ligados de algún modo con la observación) unos postulados generales de los que derivar la teoría como un conjunto de proposiciones que tendrán también carácter general o "universal”. Estas proposiciones se deducen de esos postulados con ayuda de la lógica, esencialmente la matemática (mi presente discurso, como el de gran parte de la obra de Artigas, se refiere directamente a las teorías físicas, por lo que tienen de paradigma de las otras disciplinas científicas). Algunas de esas proposiciones describen hechos experimentalmente contrastables - dentro del ámbito de objetivación propio de la teoría - y son sometidas al control de la experiencia. Si pasan ese control, decimos que el experimento ha corroborado nuestra teoría acerca de aquel particular ámbito de la realidad natural (por ejemplo, el ámbito de los fenómenos electromagnéticos). En caso contrario, la teoría se habrá mostrado inválida como descripción de ese ámbito de la realidad natural. Ha sido "falsada” por una experiencia anámola. Se hace necesario entonces buscar nuevos postulados de los que deducir una teoría más amplia que dé cuenta también de esa experiencia, que ya no será entonces 
experiencia anómala, pues habrá quedado al abrigo de la nueva teoría. De acuerdo con esta descripción de la teoría, dos conjuntos de postulados que se implicasen mutuamente servirían como base de una misma teoría. Los postulados serían distintos, pero la teoría sería la misma. Caso más interesante es aquel en que los postulados no se implican mutuamente, y por tanto las teorías deducidas de ellos son distintas, pero tienen las mismas consecuencias observables. Los epistemólogos dicen entonces que se trata de teorías equivalentes, ya que valen lo mismo, de esta manera reconocen que lo válido de una teoría, lo que la teoría afirma acerca de la realidad, son solamente sus consecuencias observables.

Junto a la descripción del método científico está el tema de la demarcación, o incluso caracterización, de la ciencia experimental. No se trata aquí de la demarcación del conocimiento válido en general, pues, solo un positivismo gratuito -incapaz de justificarse a sí mismo- reduciría el conocimiento humano al que nos proporcionan las ciencias experimentales. Esta es la afortunada concepción de Karl Popper, quien pone como criterio de demarcación la posibilidad de falsación, algo que, a grandes rasgos, es universalmente admitido, al menos implícitamente (Incluso por quienes niegan esta posibilidad a corto plazo - como Kuhn o Feyerabend-, pues, a largo plazo, nadie en realidad la negaría, como ya he argumentado). Si se intenta dar un paso más y proponer incluso una caracterización del método científico, la propuesta objetivista de Mariano Artigas y Evandro Agazzi -que caracterizan este conocimiento por el hecho de crear su propio objeto de estudio- lo distingue de la filosofía como búsqueda de verdad. En efecto, la filosofía mira a la realidad tal cual es, no sometida a un particular proceso de objetivación, como realidad ad hoc para un determinado estudio.

Entre las estipulaciones instrumentales o criterios de protocolaridad con que es creado el objeto de una teoría científica, Mariano Artigas incluye los postulados de la teoría como "estipulaciones axiomáticas que determinan qué enunciados se toman como axiomas de la teoría” (Artigas 1999, 276). El objeto de una ciencia queda así definido como aquello que cumple esos postulados. 
Este punto de vista tiene la ventaja, a mi parecer, de que explica la claridad del método científico, ya que convierte a la ciencia en el estudio de lo que uno mismo ha definido mediante la proposición de postulados. Se explica entonces por qué ha resultado suicida para la filosofía la exigencia racionalista de "ideas claras y distintas", pues esto solo puede proveerlo el método científico ${ }^{3}$.

La concepción construccionista de la ciencia de Artigas y Agazzi no está abocada al convencionalismo porque, aunque haya puesto los postulados entre los convenios de la teoría, no los considera como mero convenio.

¿Son los postulados tan solo estipulaciones? Esta es la pregunta clave, cuya respuesta nos situaría en una versión epistemológica del apriorismo kantiano o del hilemorfismo aristotélico, predisponiéndonos hacia a una $\mathrm{u}$ otra postura en el terreno filosófico ${ }^{4}$. Con esta pregunta se inquiere si hay pretensión de verdad en los postulados, es decir en el implicante de $p \rightarrow l$. La negación de verdad, o de pretensión de verdad, en $l$ lleva necesariamente a esta misma negación en $p$. Por tanto, cuando los convencionalistas (Mach, como paradigma) o meramente conjeturalistas (Popper) niegan pretensión de verdad en la teoría, la están negando también en los postulados: para Mach serían solo convenciones, y para Popper serían convenciones que además pretenden decir algo acerca de la realidad pero solo de modo conjetural.

3 Se hace entonces particularmente claro que Guillermo de Ockham fue, en el fondo, el primer filósofo moderno. En su Summa totius logicis trata principalmente de los universales, pero entendidos estos como mera construcción humana, cuando se escogen unos caracteres comunes en diversos seres, y al juntarlos se crea un concepto aplicable a todos ellos. En realidad, esto es exactamente lo que sucede en las teorías científicas, en las que se construyen, mediante definición, los conceptos que se quiere estudiar. No es casualidad que los primeros conceptos físicos construidos mediante definición -velocidad, movimiento uniforme, movimiento uniformemente acelerado, velocidad media- hayan sido definidos en Oxford y a principios del siglo XIV, precisamente en el lugar y tiempo de formación de Guillermo de Ockham. De hecho, el estudio de la cinemática - preludio de la física- floreció en las escuelas de filosofía nominalista. La emulación del método científico en el método filosófico, rasgo característico de la filosofía de la Edad Moderna, arranca pues de los inicios mismos de la ciencia física en el tardomedievo.

4 Aunque no la implique, pues epistemología y gnoseología son discursos distintos, ya que el conocimiento científico constituido por predicciones falsables es muy distinto del conocimiento ordinario donde no se dan tales predicciones (a menos que uno crea en el tarot). 
Ambos son, pues, realistas ontólogicos pero escépticos gnoseológicos (o más propiamente epistemológicos), aunque en menor grado Karl Popper.

Se puede negar la verdad, o pretensión de verdad en el implicante $p$ de $p \rightarrow l$, sin por ello negarla en el implicado $l$. Una postura así, convencionalista en los postulados pero realista en la teoría o conjunto de leyes, podría ser articulada según la antigua distinción del objeto formal de una ciencia: el objeto formal quod, es decir el objeto que la ciencia estudia, es decir, la parcela de la realidad natural objetivada por unos determinados protocolos operacionales de experimentación; y el objeto formal quo, es decir el objeto con que se realiza el estudio, que serían aquí los postulados de la teoría. Los postulados no serían entonces lo que se estudia, sino el modo en que se estudia algo que se conjetura que cumple los postulados en el nivel de aproximación propio de la teoría, pero que no viene definido por ellos.

La afirmación de verdad en el implicado $l$ de $p \rightarrow l$ lleva a la afirmación de verdad en el implicado. Por tanto el constante posicionamiento de Artigas en favor del valor cognoscitivo de la teoría científica como conocimiento de la realidad, aunque parcial (ámbito objetivado) y aproximado (escala de la objetivación) y por tanto provisional (el ámbito es ampliable, la aproximación es mejorable), no implica posicionamiento alguno sobre la pretensión de realidad de los postulados. Sin embargo, permite adivinar su respuesta a la que he llamado pregunta clave: los postulados son estipulaciones de la teoría, sí, pero no solo estipulaciones sino que hay en ellos pretensión de verdad (errónea o no) acerca de la realidad natural, en el ámbito y escala de una particular objetivación. Justificar esta respuesta supondrá mostrar la racionalidad de la inferencia de postulados desde leyes universales experimentales (remontando $p \rightarrow l$ ), y esto después de haber justificado la inducción de estas leyes desde los hechos experimentales particulares (remontando $l \rightarrow h$ ).

Leída en su integridad la obra principal de los autores citados, Ernest Mach (La ciencia de la mecánica), Pierre Duhem (La teoría física), Karl Popper (La lógica de la investigación científica) y el mismo Evandro Agazzi (Temi e problemi di filosofía della física), el lector no encontrará estas justificaciones. En cambio, sí encontrará en cada una de ellas expresiones de admiración 
por el hecho de que la ciencia tenga éxito, pero no la común admiración por lo impresionante de sus avances, sino otra de índole más filosófica, que radica en el hecho de que su epistemología no ha dado una explicación - una justificación racional- de la fiabilidad del método que con tanta precisión describen. Se trata más bien del asombro por algo que en su voluminosa obra no han llegado a explicar (ique me devuelvan el tiempo y el dinero!). Pierre Duhem es consciente de este hecho ${ }^{5} \mathrm{y}$ añade a sus propias consideraciones citas de Ernst Mach (La ciencia de la Mecánica) y de Henri Poincaré (La science et l'hypothèse) - los autores que sigue más de cerca- en las que muestran su asombro por el progreso logrado con un método cuya fiabilidad no han llegado a fundamentar. A ellos se refiere Pierre Duhem cuando afirma en el capítulo IV de La teoría física: "Los esfuerzos encaminados a lograr una teoría física cada vez más unitaria y más perfecta son razonables, aunque la crítica del método físico no haya podido descubrir la razón”.

En los Temi e problemi di Filosofia della fisica de Evandro Agazzi, el autor se excusa de tratar el tema de la inducción porque sería demasiado extenso. Remite entonces a la obra de otro autor, C. Hempel, de hecho epistemólogo anti-inductivista. En la obra de Popper, la inducción se trata como un tipo de proposición no falsable, y por tanto no científica, por lo que se siente excusado de discutirla. En cuanto a la obra de Thomas Kuhn sobre la estructura de las revoluciones científicas, la discusión de la inducción en su obra, o mejor dicho la ausencia de esta discusión, es despachada con un “en algún rincón de este laberinto debe agazaparse, por ejemplo, el problema de la inducción".

A mi entender, la ausencia de esta discusión de importancia capital para la fiabilidad del método científico se debe a un error de método precisamente en autores que tratan del método. Esto resulta especialmente palmario en Karl Popper, quien no distingue entre la descripción del método científico y la discusión de la fiabilidad. El debate sobre la validez de la inducción se sitúa en el segundo plano, y no es por tanto cuestión científica sino filosófica, y por tanto su afirmación no tiene por qué pasar un criterio de falsabilidad. 
En una obra de epistemología debe discutirse la fiabilidad del principio de inducción, pero sin los estándares del método experimental, sino con los propios de cualquier discusión filosófica, como podría ser la fundamentación del derecho (Y si, por el hecho de no ser científica, vemos un sinsentido en esta discusión, estamos adoptando ya una actitud filosófica - concretamente positivista - y la adoptamos sin fundamentación alguna. Dejamos así a la ciencia misma sin fundamento).

\section{Lógica y racionalidad}

Como preámbulo a esta discusión, distingamos claramente entre lógica y razón, la segunda incluye mucho más que la primera. Con solo lógica no se puede llegar a la verdad de proposición alguna, sino solo al hecho de que de unas proposiciones se deducen otras. Las funciones lógicas básicas están implementadas en cualquiera de nuestros ordenadores, y no por ello decimos que se trata de un ser racional. Aunque en el pasado la palabra “demostración” tuvo un sentido más amplio, en la actualidad, a consecuencia de su precisa definición en lógica simbólica, tiene más bien la connotación de

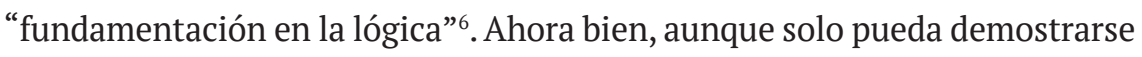

${ }^{6}$ Un claro ejemplo de este cambio de sensibilidad en torno al significado de la palabra “demostración" afecta a la posibilidad de "demostrar la existencia de Dios”. Descartes, por ejemplo, no duda en presentar su filosofía como una demostración de la existencia de Dios, palabra a la que el hombre actual es alérgico, debido al significado preciso que esta palabra ha recibido en la lógica formal (hasta el punto de que la demostración, tal como hoy la entendemos, solo se da en matemática -y en las ciencias en que la matemática es aplicada- para inferir una proposición de otras proposiciones). Zubiri, por ejemplo, en "Naturaleza, Hombre, Dios", prefiere hablar de “acceso intelectual a la divinidad". Se trata pues de argumentos para mostrar una verdad, más que "demostrarla”. En el Tractatus Logicus Philosophicus Wittgenstein termina reconociendo que, de acuerdo con la propia obra, nada lo que ha escrito tiene sentido, pero ha servido para "mostrar" lo que quería decir: "Mis proposiciones esclarecen porque quien me entiende las reconoce al final como absurdas, cuando a través de ellas - sobre ellas - ha salido fuera de ellas. (Tiene, por así decirlo, que arrojar la escalera después de haber subido por ella)". Pero su lector no puede dejar de preguntarse: si esta obra, sin ser reducible a pura lógica, a pura demostración, ha podido "mostrarme" cierta realidad, ¿por qué no pueden hacerlo también las argumentaciones propias del saber filosófico, por ejemplo las argumentaciones sobre la existencia de Dios? Lo mismo puede decirse de las demás filosofías que yo llamaría "de la escalera", tan abundantes en la literatura moderna - por ejemplo, el Curso de Filosofía Positiva de 
en este sentido que unas verdades implican otras - sin que pueda demostrarse, en este sentido estricto, verdad alguna- bien puede ser mostrada racional, fundamentada en la razón, la verdad de nuestras afirmaciones. No es lo mismo algo afirmado sin razón alguna que algo para lo que se aportan razones, aunque no se trate de una "demostración" en el sentido estricto de la lógica. Si alguien intentara negar que soy hijo de Josefina Lucía, por ejemplo, tendría yo un buen cúmulo de razones para afirmar que sí lo soy, pero si persistiera en esa tontería, yo podría pedir un análisis de su ADN y el mío: que fuese comparada esa sucesión de millones de letras A, T, C, G (los nucleótidos Adenina-Timina, Timina-Adenina, Citosina-Guanina, Guanina-Citosina) en que consiste nuestro genoma. No se analizan todos, obviamente, en las pruebas de ADN, pero supongamos que esta vez se procediera así. Si apareciera que en cada par de cromosomas míos hay uno cuyos ciento cincuenta millones de nucleótidos están secuenciados exactamente como en un cromosoma de mi madre, se habría "mostrado" que soy hijo de ella, pero no se habría "demostrado" en el sentido lógico de la palabra, puesto que "lógicamente” es posible que la coincidencia haya sido casual.

Para un joven David (David Hume) que, lanzando su piedra contra Goliat, mantenga que la maternidad de mi madre, aun después del resultado positivo de esta prueba exhaustiva de ADN, no ha alcanzado justificación racional, nada de lo que yo diga en adelante como justificación del método científico - y en particular de la inducción- será racional. Pero creo que ese joven David andará confundiendo racionalidad con lógica, al interpretar la ausencia de demostración lógica con ausencia de racionalidad. Si ha de ser consecuente con su propio discurso, solo podrá justificar racionalmente el método matemático, precisamente la ciencia en la que nada se afirma

Comte-, que al desautorizar la argumentación filosófica en general, se desautoriza a sí mismas. David Hume escribe al final de su Investigación sobre el entendimiento humano: "Cuando recorremos las bibliotecas, persuadidos de estos principios, ¡qué estragos no haremos! Si tomamos en nuestras manos un volumen de teología o metafísica escolástica, por ejemplo, preguntémonos: ¿contiene algún razonamiento abstracto sobre la cantidad y el número? No. ¿Contiene algún razonamiento experimental sobre cuestiones de hecho y de experiencia? No. Arrójese entonces a las llamas, pues nada puede contener sino sofistería y engaño". Al lector le queda la idea de que, si toma esto en serio, también debería arrojar al fuego el libro que tiene entre las manos. 
acerca de la naturaleza, como bien observa Wittgenstein en su Tractatus. En efecto, en la matemática tan solo se afirma que los enunciados que llamamos teoremas son deducibles de los diez axiomas de la teoría de conjuntos ZFC (Zermelo-Fraenkel with choice, dejando la hipótesis del continuo como un tecnicismo que solo interesa a los lógicos).

Veamos si hay justificación racional de mi filiación respecto de Josefina Lucía tras el resultado positivo de la prueba. En primera aproximación, "racionalidad" significa que tenga razones para afirmar algo: en este caso, mis propios recuerdos de infancia, el testimonio unánime de muchas personas, nuestro evidente parecido, y, al final, esta supuesta prueba del ADN realizada con éxito sobre la totalidad de nuestro genoma. Pero en segunda aproximación, cuando el joven David me pregunte qué significa “tener razones” para afirmar algo, diré que al justificar mi filiación he sido racional porque he hecho "lo que la razón siempre hace". ¿Y qué es lo que hace siempre la razón? Busca la explicación más sencilla. Este lugar común en la epistemología moderna queda bien ilustrado por el descubrimiento de las leyes de Kepler: El matemático de Gratz encontró que todas las posiciones del planeta Marte observadas por Tycho Brahe estaban sobre una elipse, y concluyó que esa elipse era su trayectoria. En realidad, muchas otras curvas pasan también por esas posiciones, pero ni se le ocurrió pensar en ellas: puso la elipse por ser la más sencilla de todas. Popper tratará el tema de la explicación más sencilla, propuesto antes por Poincaré, diciendo que es también la más sencilla de mostrar falsa, y por eso la ve como la "más fácilmente falsable”, y por tanto la explicación más científica. Sea como fuere, es comúnmente admitido que esto hace siempre la razón: pone la explicación más sencilla. Evidentemente, hay otras explicaciones para esos hechos que muestran - aunque no demuestran-que soy hijo de mi madre: es posible que todas las personas que me rodean se hayan conchabado para hacerme creer desde pequeño que yo era su hijo: familiares, médicos, vecinos... Es lógicamente posible que mi parecido con ella sea meramente casual... Es lógicamente posible que la secuenciación de millones de nucleótidos haya coincidido porque en realidad mi ADN fue sintetizado artificialmente en un laboratorio cuando yo nací, a vista de la secuencia 
del ADN de Josefina Lucía. Si protesto a esto diciendo que en el año 1949 en que yo nací, ni siquiera se conocía el ADN, puesto que lo descubrieron Watson y Cricks en 1953, el joven David puede decir que bien pudo haber sido descubierto antes de 1949 y haber sido secuenciado el genoma humano, y aprendido a sintentizar, pero mantenido en secreto hasta ahora para gastarme a mí este bromazo (cosas así creen los locos, a quienes falta la razón, sí, pero no la lógica). Todo esto es lógicamente posible, y nadie podrá "demostrar" su imposibilidad, pero no es razonable. Al menos David debe admitir que la maternidad de Josefina Lucía es más razonable que todas las explicaciones ad hoc que él tiene que inventar contra cada una de las evidencias que yo aporto. Son tan artificiales, tan complicadas e increíbles, que resulta mucho más sencilla mi explicación de todas esas coincidencias por un solo hecho: Josefina Lucía es mi madre. Si ni aun con esto el joven David quedara callado, y mantuviese aún que la coincidencia de los tres mil millones de nucleótidos ha podido ser casual, yo observaría entonces que su explicación de la coincidencia en la secuenciación del ADN es aún más artificial e increíble que aquel increíble bromazo.

Pero nuestro radical David aún puede argumentar algo, y conviene ser sumamente escrupuloso en este análisis, atentos a lo certero de su posible objeción: en vez de escribir los caracteres genómicos como A, T, C, G, escribámoslos como 1, 2, 3, 4. Mi genoma, y el de Josefina Lucía, será pues una sucesión de unos tres mil millones de dígitos, siendo cada uno de ellos 1, 2, 3 o 4. Por tanto, la sucesión de diferencias entre ambas sucesiones de nucleótidos, los míos y los de Josefina Lucía puestos en comparación, serán tres mil millones de dígitos, cada uno de ellos pudiendo valer 3, 2, 1, 0, -1, $-2,-3$. Al decir que ambos, mi madre y yo, tenemos igual (la mitad de) nuestro genoma, lo que estamos diciendo es que esa sucesión de diferencias consiste en tres mil millones de ceros. La objeción de David sería: ¿Por qué esta sucesión de dígitos no puede ser explicada por la mera casualidad, y, sin embargo, cualquier otra sucesión de diferencias de dígitos entre el 3 y el -3 sí puede ser explicada por la casualidad, siendo así que ambas sucesiones tienen la misma probabilidad? La objeción no es en modo alguno baladí, y en el fondo es la que ponen cuantos niegan la racionalidad del principio de 
inducción (la coincidencia de los dígitos en el ADN se tornará en este caso en coincidencia de los resultados de las experiencias repetidas hasta ahora, coincidencia de la que, a su entender, nada se puede concluir en estricta racionalidad). Por eso me parece un error centrarse en la probabilidad (todo lo que hasta ahora se ha dicho a favor de la inducción, en epistemología), puesto que, en efecto, esa sucesión de tres mil millones de ceros no tiene menor probabilidad que cualquier otra sucesión de diferencias.

La idea que quiero sugerir en este artículo en honor de nuestro querido epistemólogo es que para contestar a David con algo bien cortado, hay que hablar de la entropía de la sucesión, concepto definido con precisión en la teoría de la complejidad de Kolmogoroov-Chaitin ${ }^{7}$. Esta entropía mide el grado de desorden que se da en la sucesión (minimizar la entropía, pues, es aumentar el orden). En el grado de entropía sí son distinguibles ambas sucesiones, pues la primera, la sucesión 0000..., tiene una entropía bajísima -un muy alto grado de orden-, mientras que otra, la que suponemos tomada al azar, no tiene por qué tenerla. En realidad, no es la baja probabilidad de la secuencia de diferencias $0000 .$. lo que requiere una explicación (pues

7 Se define el grado de complejidad de Kolgomorov-Chaitin, o entropía algorítimica (yo diré simplemente "entropía”) de una sucesión de dígitos (escribámosla como una sucesión de unos y ceros) como el menor número de dígitos que se requieren para definir una máquina o algoritmo que la generen. Si no existe tal algoritmo, salvo escribir la propia sucesión, se le llama "de azar", según la definición de Chaitin de sucesión de azar. En el caso que nos ocupa sí que existe tal algoritmo y de descripción muy breve: "tres mil millones de dígitos, el primer dígito es cero, y cualquier otro dígito es igual al anterior” Al escribir esta frase como sucesión de ceros y unos, necesitaremos un número de dígitos $\mathrm{n}$ menor que tres mil millones de dígitos, por lo que esta sucesión no es de azar, sino que, al describirla de este modo, hemos encontrado cierto orden, cierta forma, en ella que hemos utilizado para describirla: hemos minimizado (matemáticamente "hemos acotado superiormente") su entropía. Pero puedo dar una descripción aún más breve: "tres mil millones de dígitos iguales a cero" Esta descripción consiste de un número $m$ aún menor que $n$, por lo que he encontrado más orden, más forma, más simplicidad en la ley de formación, que en aquella sucesión: la acotación de entropía ha sido más fuerte, o en palabras equivalentes, hemos minimizado aún más la entropía de la sucesión. El apriorista kantiano dirá que yo he puesto esa forma en la sucesión, pero el hile-morfista aristotélico afirmará que esa forma estaba ya en la sucesión y yo la he encontrado. Esto es lo que diferencia apriorismo e hilemorfismo: ambos hablan de forma en el conocimiento, pero uno la pone como apriorismo de nuestra facultad de conocer, y el otro la ve en la realidad. Ambos dicen que entender es poner orden en lo que antes parecía inconexo, pero uno dice que ese orden lo hemos puesto nosotros y el otro dice que ese orden estaba ya y lo hemos encontrado. 
es tan baja como la probabilidad de cualquier otra sucesión de diferencias) sino la baja entropía de esta sucesión de diferencias. Una sucesión de la más alta entropía posible -el mínimo orden posible-es una sucesión de azar, definida esta por Chaitin como una sucesión que no puede ser generada por una máquina o algoritmo para cuya definición se requieran menos dígitos que los que tiene la sucesión misma, es decir una sucesión cuya información sea incompresible por no tener ninguna ley de formación: una sucesión en la que no se da ningún orden. Evidentemente, la sucesión formada por tres mil millones de ceros no es en modo alguno sucesión de azar, pues el algoritmo que la genera es de simplicísima definición: "escríbase el dígito 0 tres mil millones de veces".

\section{Justificación de la inducción de leyes generales}

Los enunciados de leyes generales implican el enunciado particular de cada hecho experimental, pero no al revés: el hecho de que un material se haya dilatado con el calor en experiencias particulares, y además lo haya hecho siempre con el mismo coeficiente de dilatación, no implica, en estricta lógica, que ese material se dilate con el calor y con ese coeficiente, como ley general, puesto que lo particular no implica nunca lo universal. Sin embargo, se llega por inducción a leyes generales desde enunciados particulares de experiencia, y trataré ahora de su validez. Este tema tiene alguna resonancia filosófica, pues todo nuestro conocimiento, no solo el científico, procede en cierto modo por inducción ya que solo observamos hechos particulares, pero expresamos nuestro conocimiento en términos universales. Por tanto, desprovista de racionalidad la inducción (tanto en gnoselogía como en epistemología), desprovista también de racionalidad nuestra propia razón, como pez que se muerde la cola. Algo así ha pasado en filosofía: la estricta exigencia de racionalidad (en la "philosophie mathematique" racionalista, según la expresión de Descartes) ha dado lugar, al final de la jornada, a las llamadas “filosofías de la sospecha”. Pero no excedamos nuestros objetivos y restrinjámonos a la inducción epistemológica. Sostengo que sí es racional, porque hay razón para enunciar la ley general, o al menos hay más razón 
que para no enunciarla, y porque al proceder por inducción hacemos lo que la razón siempre hace.

En efecto, la explicación más sencilla de que hasta ahora siempre se haya dilatado este material con el calor y siempre con el mismo coeficiente de dilatación (supuestas las mismas condiciones) es que necesariamente tenía que ocurrir así, aunque no supiéramos aún por qué (en la actualidad lo sabemos, pues la dilatación por el calor, e incluso los coeficientes de dilatación, se pueden deducirse actualmente con ayuda de la mecánica cuántica, en teoría del estado sólido). La explicación contraria, que haya sucedido siempre así y con el mismo coeficiente de dilatación no por razón necesaria sino por mera casualidad, es tan artificial e increíble, aunque lógicamente posible, como el bromazo organizado para hacerme creer que Josefina Lucía es mi madre. $\mathrm{O}$ al menos, esa explicación no debe ser considerada como más racional que nuestra la afirmación de que, si se ha obtenido siempre el mismo resultado en tantas experiencias, es porque necesariamente debía obtenerse ese resultado, aunque ignorásemos se ignorara entonces - antes del advenimiento de la mecánica cuántica- la razón de esa necesidad. Al proceder así, procedemos como lo hace siempre la razón, pues tomamos la explicación más simple de los hechos, y rechazamos las explicaciones más complicadas, como que la repetida coincidencia experimental se haya debido a un cúmulo de casualidades. (Si no procediésemos así, ni siquiera usaríamos la razón. No habría nada que aunar, nada que relacionar, ningún orden o forma que encontrar en los datos que nos llegan: todo serían hechos inconexos, coincidencias, pura casualidad). Hay pues fundamentación racional para la inducción de leyes generales, aunque no haya fundamentación lógica pues la lógica no puede ascender de implicado a implicante (Pero esto último no significa que la inducción sea ilógica, como frecuentemente se ha dicho, pues ambos implicado e implicante son compatibles, es decir, pueden ambos ser verdad al mismo tiempo). La clave aquí ha sido nuestra distinción inicial: la razón es más que la lógica, lo racional es más que lo meramente lógico.

Se podría objetar que mi justificación entrópica de la inducción (y por tanto, en cierto modo, del conocimiento humano) no va más allá de la 
concepción de Ernst Mach en La ciencia de la Mecánica, cuando dice que la inducción científica de leyes generales y, desde estas, la inducción de postulados no aporta nuevo conocimiento puesto que solo pone orden en los datos que recibimos, como quien ordena una biblioteca. Yo digo que sí aporta conocimiento, puesto que ese orden es forma que está en los datos (lo que negaría el apriorista kantiano). El conocimiento encuentra ese orden, esa forma (como diría el hilemorfista), como noticia recibida de la naturaleza.

Un análisis más detenido nos lleva a ver en cada ley experimental dos enunciados: Uno que afirma que hasta ahora siempre ha sucedido así, que las muestras de ese material siempre se han dilatado con el calor y con ese coeficiente; y otra que afirma que siempre va a suceder así en el futuro. La primera es una proposición; la segunda es una predicción.

La primera, la proposición, puede ser verdadera o falsa. De hecho es una conjunción de muchas proposiciones particulares que afirman la dilatación de cada muestra de material con que se ha experimentado, y, en cuanto tal, su verdad se deduce en estricta lógica de la verdad de cada una de esas proposiciones. Lo que afirmamos es que se ha encontrado una ley que se ha cumplido en todas esas experimencias: se ha encontrado un cierto orden que estaba en todas ellas, no que yo lo haya puesto. Se ha minimizado, pues, la entropía de los datos de experiencia al encontrar ese orden o ley en ellos.

La segunda, la predicción, no puede ser verdadera o falsa, sino que puede cumplirse o no cumplirse. Pero por el modo en que hemos llegado a formularla, puede ser razonable o puede no haber razones para sostenerla (aunque no cabe duda de que ambos, el cumplimiento de la predicción y la racionalidad de su formulación, están estrechamente relacionados). Es interesante la observacion de Popper, que se encuentra también en Agazzi, de que no se tiene noticia hasta el presente de una sola ley obtenida por inducción por constancia de resultados experimentales -en un determinado nivel de aproximación y en un determinado ámbito de experimentación-, que haya debido cambiarse porque de pronto la naturaleza haya empezado a comportarse de modo distinto (Aunque sí ha habido leyes, por supuesto, que han resultado inválidas al mejorar el nivel de aproximación, o al introducir nuevos factores en el ámbito de experimentación, como en el experimento 
de Michelson-Morley). Así pues, el problema de la inducción no consiste en explicar por qué ha fallado (en una experiencia anómala que haya dado lugar a la búsqueda de una nueva teoría), sino en explicar por qué nunca ha fallado, en un mismo ámbito y a un mismo nivel de aproximación. No ha fallado porque la inducción ha sido un proceso racional en cuanto ha tratado de lo ocurrido y un proceso razonable en cuanto ha predicho lo que iba a ocurrir. La inducción tenía la razón a su favor. Es la razón la que no ha fallado.

\section{Justificación de la inferencia de postulados}

Pasemos ahora a fundamentar este otro tipo de inducción, aunque llamada así de modo impropio, en que la implicación $p \rightarrow l$ es remontada. Es un tema de menor envergadura filosófica que la inducción propiamente dicha, pues no tiene paralelo en nuestro modo ordinario de conocer. Ni siquiera tiene un paralelo en el resto de las ciencias experimentales, pues cuando se habla en ellas de postulados se hace metafóricamente, por vía de analogía con las teorías de la física.

Quienes, como Ernst Mach o Karl Popper, no ven en los postulados más que meras convenciones, o meras conjeturas acerca de la realidad, esta justificación no es necesaria pues quien solo conviene o conjetura, nada afirma. Y quien nada afirma, nada necesita fundamentar. Pero sí resulta necesaria para quienes, como Isaac Newton y de hecho la inmensa mayoría de los científicos, ven los postulados como verdaderamente descriptivos de la realidad natural, dentro de un determinado ámbito y nivel de aproximación, y por tanto provisionales. Dirigido a ellos, mi discurso, mi argumento será el mismo que en el caso de la inducción de leyes generales: la proposición de postulados es racional porque hay razón para ello - mayor razón que para rechazarlos-, y también porque procedemos del modo en que siempre lo hace la razón. Si unos pocos postulados implican una multitud de resultados observables en la experimentación que se cumplen siempre con precisión asombrosa, tal como ocurre en cada una de las mecánicas, dentro de su ámbito y nivel de aproximación, parece inadmisible atribuir ese cumplimiento a la casualidad, en vez de atribuirlo a la verdad de los 
postulados (La palabra observable es aquí importante pues los postulados mismos pueden no serlo, como los postulados de la mecánica cuántica, ya que utilizan la unidad imaginaria).

Se ha escogido así, de nuevo, la explicación más simple para hechos que de otro modo aparecerían inconexos: una complicada e increíble acumulación de casualidades que haría de cada una de esas leyes su propio postulado, todas ellas sin mutua conexión. Hemos hecho, pues, lo que la razón siempre hace: buscar la explicación más simple. No poner forma, sino encontrar forma allá donde estaba.

Eso no significa que la razón humana sea infalible, pues la explicación encontrada puede no ser la más simple, o puede la explicación más simple no ser la verdadera. Infalible solo es la lógica, es decir la matemática, aunque a costa de no decir nada acerca de la realidad. Sin embargo, hay que complementar esta afirmación prudencial con la mención de que tal fallo nunca se ha dado en la historia de la ciencia física, entendida esta desde el momento en que se dedujo la mecánica clásica a partir de unos axiomas (Philosophie naturalis principia matematica. Newton, 1687). Nunca ha sucedido que después de haber desarrollado una teoría física con base en unos postulados, haya habido que cambiarlos luego por haber resultado erróneos, sin que haya cambiado el objeto mismo de la teoría por aparición de un fenómeno nuevo que amplíe el ámbito de aplicación de la teoría (el caso de la experiencia de Michelson-Morley, la radioactividad, o la radiación de cuerpo negro), o por una mejora en el nivel de aproximación en los instrumentos de medida. De nuevo, el problema de la fiabilidad no es pues explicar por qué las teorías han fallado, sino explicar por qué las teorías nunca han fallado. Y la respuesta es, de nuevo, que no han fallado porque se han basado en la razón humana (no en la lógica, que es impersonal). De nuevo, es la razón humana la que no ha fallado.

Vemos aquí, de modo aún más claro que en el caso de la inducción, cuánta equivocación hay en intentar fundamentar la ciencia en la lógica, por supuesto con resultado negativo. Y es que la lógica es atemporal, nunca tomará en consideración el tiempo. Sucede como en la observación de Heidegger en Ser y Tiempo: la lógica, por su atemporalidad, nunca podrá 
atrapar el sentido del ser, porque el sentido del ser es su temporalidad y por tanto ha de ser conocido a lo largo del tiempo. Análogamente, podemos decir aquí que la lógica nunca podrá fundamentar la ciencia, porque la ciencia es logro del hombre a lo largo del tiempo, y la lógica es ciega a él. Me explicaré mejor. Todos sabemos que es esencial para la justificación de una nueva teoría el cumplimiento de sus predicciones. Por ejemplo la teoría del Big Bang predice una radiación de fondo de 2’7 grados Kelvin ¡y así se ha encontrado! La teoría de partículas predice aquellas que luego se han encontrado exactamente con las características predichas. La teoría electrodébil, según aparece en el modelo estándar de la gran unificación, predice la corriente neutra y el bosón de Higgs que han aparecido tal como se esperaba. El cumplimiento de las predicciones es el espaldarazo para las hipótesis sobre la naturaleza. Sin embargo, desde el punto de vista de la lógica, que no ve el tiempo, en igual situación se hallan, respecto de las hipótesis, las predicciones cumplidas que las experiencias explicadas (pues la diferencia entre ambas categorías es temporal): ambas son implicadas por la hipótesis pero no la implican. La lógica es, pues, inadecuada para justificar la fiabilidad de los postulados de las teorías científicas. Pero la razón sí justifica esta fiabilidad. La inducción de leyes generales desde hechos experimentales, y la inferencia de postulados desde esas leyes generales no es un proceso lógico, pues, pero sí un proceso racional.

Una última observación, ya de interés puramente filosófico. Puesto que hemos encontrado fundamentación racional - no fundamentación lógica-para los postulados de las teorías científicas con base en las leyes experimentales de que se dispone, lo propio es decir que se trata de juicios a posteriori, es decir basados de algún modo en la experiencia. Quienes ven en estos postulados meras convenciones o definiciones, y quienes ven en ellos meras conjeturas, ni siquiera los entienden como juicios. Por otra parte, los juicios de la base experimental de la ciencia son juicios a posteriori (incluidas las leyes generales obtenidas por inducción, puesto que esta inducción está racionalmente justificada a partir de los hechos de experiencia); y los juicios en la fase teórica -el desarrollo de la teoría desde sus postulados - son juicios analíticos, pues proceden de un análisis 
de los postulados que se han puesto como base de la teoría. ¿Dónde pues están los juicios sintéticos a priori que para Kant articulan el edificio de una ciencia? En realidad, casi nadie hoy admite que los postulados de ninguna teoria científica -las leyes de Maxwell, por ejemplo-puedan ser apriorismos de nuestro conocimiento, y por tanto tampoco lo son las leyes de ellos deducidas. La Crítica de la Razón Pura queda así comprometida, en su misma base, por nuestra actual concepción de la ciencia.

\section{Referencias}

Agazzi, E. 1969. Temi e problemi di filosofia della fisica. Milano: C. Manfredi Editore. Artigas, M. 1992. Ciencia y fe. Nuevas perspectivas. Pamplona: Eunsa.

-. 1994. El desafío de la racionalidad. Pamplona: Eunsa.

-. 1999. Filosofía de la ciencia experimental. La objetividad y la verdad en las ciencias. Pamplona: Eunsa.

Comte, A. 1981. Curso de Filosofía Positiva. Buenos Aires: Aguilar.

Duhem, P. 2003. La teoría física: su objeto y su estructura. Herder: Barcelona. Originalmente publicado como Duhem, P. 1914. La théorie physique. Son objet, sa structure. Paris: Vrin.

Feyerabend, P. K. 1989. Contra el método. Esquema de una teoría anarquista del conocimiento. Barcelona: Ariel. Originalmente publicado como Feyerabend, P. K. 1974. Against Method. Outline of an Anarchistic Theory of Knowledge. Minneapolis: University of Minnesota.

Heidegger, M. 1967. Ser y tiempo. México: FCE. Originalmente publicado como Heidegger, M. 1927. Sein und Zeit. Tübingen: Max Niemeyer Verlag.

Heisenberg, W. 1971. Physique et philosophie. Paris: Éditions Albin Michel. Originalmente publicado como Heisenberg, W. 1958. Physics and phylosophie. New York: Harper \& Brothers.

Hume, D. 2004. Investigación sobre el entendimiento humano. Madrid: Istmo. Originalmente publicado como Hume, D. 1748. An Enquiry concerning Human Understanding. London: A. Millar.

Kant, I. Crítica de la Razón Pura. México: Porrúa. Originalmente publicado como Kant, I. 1781. Kritik der reinen Vernunft. Riga: Johann Friedrich Hartknoch.

Kuhn, T. 1987. La estructura de las revoluciones científicas. México: FCE. Originalmente publicado como Kuhn, T. 1962. The structure of scientific revolutions. Chicago: University of Chicago Press. 
Lakatos, I. 1999. Escritos filosóficos. Matemáticas, Ciencia y Epistemología. Alianza Editorial, 1999. Madrid. Originalmente publicado como Lakatos, I. 1978. Mathematics, Science and Epistemology - Philosophical Papers, vol. 2. Cambridge, Mass.: Cambridge Univesity Press.

Mach, E. 1960. The Science of Mechanics. Chicago: The open court publishing co. Originalmente publicado como Mach, E. 1883. Die Mechanik in Ihrer Entwicklung Historisch-Kritisch Dargestellt. Leipzig: F. A. Brockhaus.

Newton, I. 1998. Principios Matemáticos de la Filosofía Natural. Madrid: Alianza Editorial. Originalmente publicado como Newton, I. 1687. Philosophiae naturalis principia mathematica. Londini: Josephi Streater.

Poincaré, H. 1968 [1902]. La science et l'hypothèse. Paris: Flammarion.

Popper, K. 1985. La logique de la découverte scientifique. Paris: Éditions Payot. Originalmente publicado como Popper, K. 1959. The logic of scientific discovery. London: Hutchinson.

Wittgenstein, L. 1997. Tractatus Logicus Philosophicus. Madrid: Alianza Editorial. Originalmente publicado en Wittgenstein, L. 1922. Tractatus Logicus Philosophicus. London: Routledge and Kegan Paul.

Zubiri, J. 1994 [1942]. Naturaleza, Historia, Dios. Madrid: Alianza Editorial. 